\title{
Electrified Methane Reforming: Understanding the Dynamic Interplay
}

Wismann, Sebastian T.; Engbæk, Jakob S.; Vendelbo, Søren B.; Eriksen, Winnie L.; Frandsen, Cathrine; Mortensen, Peter M.; Chorkendorff, Ib

Published in:

Industrial and Engineering Chemistry Research

Link to article, DOI:

10.1021/acs.iecr.9b04182

Publication date:

2019

Document Version

Peer reviewed version

Link back to DTU Orbit

Citation (APA):

Wismann, S. T., Engbæk, J. S., Vendelbo, S. B., Eriksen, W. L., Frandsen, C., Mortensen, P. M., \&

Chorkendorff, I.' (2019). Electrified Methane Reforming: Understanding the Dynamic Interplay. Industrial and

Engineering Chemistry Research, 58(51), 23380-23388. https://doi.org/10.1021/acs.iecr.9b04182

\section{General rights}

Copyright and moral rights for the publications made accessible in the public portal are retained by the authors and/or other copyright owners and it is a condition of accessing publications that users recognise and abide by the legal requirements associated with these rights.

- Users may download and print one copy of any publication from the public portal for the purpose of private study or research.

- You may not further distribute the material or use it for any profit-making activity or commercial gain

- You may freely distribute the URL identifying the publication in the public portal 


\section{Electrified methane reforming: Understanding the dynamic interplay}

Sebastian T. Wismann ${ }^{1}$, Jakob S. Engboek ${ }^{2}$, Søren B. Vendelbo ${ }^{2}$, Winnie L. Eriksen ${ }^{3}$, Cathrine Frandsen $^{1}$, Peter M. Mortensen ${ }^{3 *}$, Ib Chorkendorff ${ }^{1 *}$.

${ }^{1}$ Technological University of Denmark, 2800 Kgs. Lyngby, Denmark, ${ }^{2}$ Danish Technological Institute, 2630 Tåstrup, Denmark, ${ }^{3}$ Haldor Topsoe A/S, 2800 Kgs. Lyngby, Denmark.

Corresponding authors:* pmor@topsoe.com \& ibchork@,fysik.dtu.dk

Keywords: SMR, Electrification, Industrial catalysis, CFD, Process intensification

\section{Graphical abstract:}

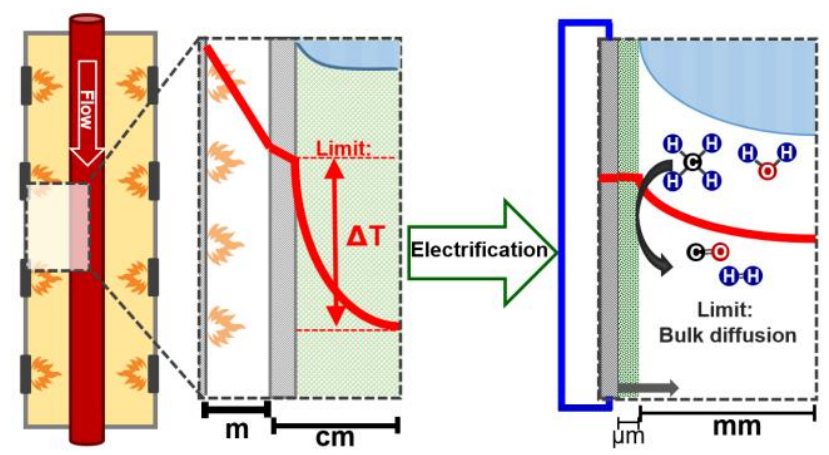




\begin{abstract}
Electrification of endothermic reactions has the potential to provide a compact and flexible reactor concept, and at the same time, substantially reduce $\mathrm{CO}_{2}$ emissions relative to combustion-heated processes. Here we show how integrated electrical heating using a wash-coated catalytic structure can resolve limiting thermal conductivity across the catalyst. The inherent uniform supply of heat enables engineering of catalytic efficiency to desired values by changing wash-coat thickness. Overall, the approach diminishes catalytic efficiency as a limiting design parameter. Instead, coat thickness will relate to catalyst lifetime, as very thin coats are more susceptible to deactivation. Characteristic timescale analysis indicates heat transfer to be the least limiting mechanism, and reactor performance is instead governed by diffusion. Optimal performance based on fluid dynamic simulations favors internal diameters below $0.5 \mathrm{~mm}$, and high linear gas velocities, towards alleviating mass transfer limitations. Integrated electrical heated steam methane reforming essentially inverts the order of reaction mechanisms compared to conventional reforming, challenging the constraints of current industrial practice.
\end{abstract}




\section{Introduction}

Constant supply of heat to catalytic sites is often limiting the efficiency of endothermic catalytic reactions. ${ }^{1}$ One of the largest endothermic processes is the production of syngas by steam methane reforming (SMR), which supplies a range of chemical industries including synthesis of; ammonia, fuels, and methanol. ${ }^{2,3}$ Reforming of natural gas and naphtha accounts for ca. $80 \%$ of the global hydrogen supply. ${ }^{3}$ Collectively, global production of syngas by steam reforming accounts for nearly 3\% of all $\mathrm{CO}_{2}$ emissions. ${ }^{2,4}$ In SMR, natural gas (methane) and steam is converted to carbon oxides and hydrogen through the overall endothermic reaction scheme in Reactions (1) and (2):

$$
\begin{gathered}
\mathrm{CH}_{4}+\mathrm{H}_{2} \mathrm{O} \rightleftharpoons \mathrm{CO}+3 \mathrm{H}_{2} \quad\left(\Delta \mathrm{H}_{\mathrm{SMR}}^{\circ}=206^{\mathrm{kJ}} / \mathrm{mol}\right) \\
\mathrm{CO}+\mathrm{H}_{2} \mathrm{O} \rightleftharpoons \mathrm{CO}_{2}+\mathrm{H}_{2} \quad\left(\Delta \mathrm{H}_{\mathrm{WGS}}^{\circ}=-41 \mathrm{~kJ} / \mathrm{mol}\right)
\end{gathered}
$$

Typical syngas conversion processes such as ammonia, methanol, and Fischer-Tropsch are facilitated at high pressure, where process economy favors operating the SMR at high pressure to decrease compression cost in the plant design. However, the SMR reaction is adversely impacted by high pressure, consequently requiring high temperatures to drive the reaction due to Le Châteliers principle (Fig. S1). Typical economic and practical operation is limited to 30 bars and $900^{\circ} \mathrm{C}$ due to material constraints. ${ }^{5}$ The high temperature requirement, low thermal conductivity, and strongly endothermic reaction, creates steep temperature gradients inside the reactor and catalyst bed, increasing the risk of carbon deposition, and cause thermal stress detrimental to reactor lifetime. ${ }^{5-7}$ The steep gradients in conventional fired reformers greatly limit catalytic efficiency, typically utilizing less than $10 \%$ of the available catalytic activity in conventional reactors. $5,8,9$

Large-scale industrial reformers can contain up to several hundred tubular reactors in a large furnace, heated by combustion of fossil fuels. ${ }^{5}$ For most large-scale reformers, radiation is the primary heat transfer mechanism. Consequently, the combustion process must occur several hundred degrees above the reaction temperature to supply the necessary heat flux to the catalyst. 5,10,11 A fired reformer typically operates near 50\% energy efficiency. Extensive heat recovery and process integration from economy of scale enable industrial plants to operate near $95 \%$ overall energy efficiency. ${ }^{12,13}$ Uniform distribution of heat is critical, favoring large furnaces. The tubular reactors are filled with catalyst pellets, optimized to provide maximum surface area with a minimal pressure drop, typically based on porous alumina impregnated with nickel.

Previous research into reducing $\mathrm{CO}_{2}$ emissions and resolving the low thermal conductivity for endothermic processes includes; new materials with higher thermal conductivity, ${ }^{14-17}$ different reactor concepts, ${ }^{18,19}$ reducing reaction temperature by shifting equilibrium, ${ }^{20-23}$ reducing characteristic length scales for heat transfer, ${ }^{24-27}$ or direct heating of the catalytic nanoparticles through induction. ${ }^{28-30}$

Integrated joule heating is a promising alternative, capable of operating with very quick start-up 
time, and practically no thermal gradients. ${ }^{31-33}$ Combined with short characteristic length scales, this conveys the potential for exceptionally small and dynamic reactors, with a well-controlled reaction front. ${ }^{33}$ With increasing implementation of renewable energy and concomitant decreasing energy costs, ${ }^{34,35}$ electrification of chemical industry is a promising, efficient, and greener alternative to the conventional fired reformers of today. ${ }^{33}$

This work describes an experimental reformer, heated by joule heating, which, supported by a detailed and verified numerical model, illustrates the benefits of electrified methane reforming. It is shown how short length scales and direct contact between heat source and catalyst changes the heat transfer dynamics and substantially reduce thermal variations compared to fired reformers. By extrapolating the model to industrial conditions, it is shown how the uniform heat source provides improved reaction control, extending feasible operating conditions and allows higher utilization of the carbon feedstock. Through characteristic timescale analysis, the limiting phenomena are identified, and it is illustrated how they can be alleviated to improve reactor performance substantially.

\section{Experimental setup}

Experimental evidence for the computational model was provided by testing in a resistance heated reforming setup. A $50 \mathrm{~cm}$ long FeCrAl tube (Goodfellow, outer diameter $6 \mathrm{~mm}$ ) was coated on the internal surface with a $130 \mu \mathrm{m}$ thick zirconia-based wash coat. ${ }^{36}$ Before calcination, $11 \mathrm{~cm}$ of the coat was removed from each end. The wash coat was impregnated with a nickel nitrate solution. ${ }^{37}$ The wash-coated catalyst was reduced in $200 \mathrm{Nl} \mathrm{H}_{2} / \mathrm{h}$ at $850^{\circ} \mathrm{C}$ for 4 hours in an oven, and passivated in $1 \% \mathrm{O}_{2}$ in nitrogen at $25^{\circ} \mathrm{C}$ before installation in the resistance-heated setup. The catalyst was reduced in-situ prior to tests at $500-600^{\circ} \mathrm{C}$. Multiple $0.25 \mathrm{~mm}$ K-type thermocouples (Goodfellow) was spot-welded to the external surface in order to measure accurate temperature profiles during experiments (SR630 Thermocouple monitor with $10 \mathrm{M} \Omega$ Internal resistance). The reactor was heated by the joule effect, using AC current applied along the reactor through copper sockets (Fig. 1A). Input was adjusted with a vario transformer and a set of coils (Ratio = 23), transforming power supplied from ca. $220 \mathrm{~V}$ to the desired potential. By increasing the potential over the reactor, the current increases proportional to Ohm's law, and concomitantly the heat losses, heating the reactor. The resistance between reactor elements, including contact resistance between the copper sockets, where measured using a sourcemeter at 1A (Keithley 2400 sourcemeter $)$. The reactor resistance between the copper sockets was $91.0 \mathrm{~m} \Omega(41.1 \mathrm{~cm}$ between copper sockets). Contact resistance was minimized by removing the oxide layer on the contact faces between the reactor and copper sockets with sandpaper prior to mounting. The contact resistance was below $1.5 \mathrm{~m} \Omega$. The resistance of the system during experiments was calculated from the applied potential, measured by an oscilloscope (Agilent infiniiVision DSO-X 2014A), and the current, measured by a current probe (Keysight 1146B). 
A feed mixture of $\mathrm{CH}_{4}, \mathrm{H}_{2} \mathrm{O}$, and $\mathrm{H}_{2}(30 / 60 / 10)$ was preheated to $105^{\circ} \mathrm{C}$ to avoid condensation. Hydrogen was included to prevent oxidation of the nickel catalyst and deposition of carbon near the inlet, equivalent to industrial practice. Steam was removed from the product by a drain trap (Armstrong 11LD). Dry gas composition was analyzed by gas chromatography (Agilent 7890 GC System with TCD/FID), with at least 4 consecutive measurements at each set of conditions. The outlet pressure was slightly above ambient ( $<50$ mbar).

The methane conversion was evaluated by the mole fractions of carbon-containing species as normalized from the GC measurements:

$$
\mathrm{X}_{\mathrm{CH}_{4}}=\frac{\mathrm{y}_{\mathrm{CO}}+\mathrm{y}_{\mathrm{CO}_{2}}}{\mathrm{y}_{\mathrm{CH}_{4}}+\mathrm{y}_{\mathrm{CO}}+\mathrm{y}_{\mathrm{CO}_{2}}} \cdot 100 \%
$$
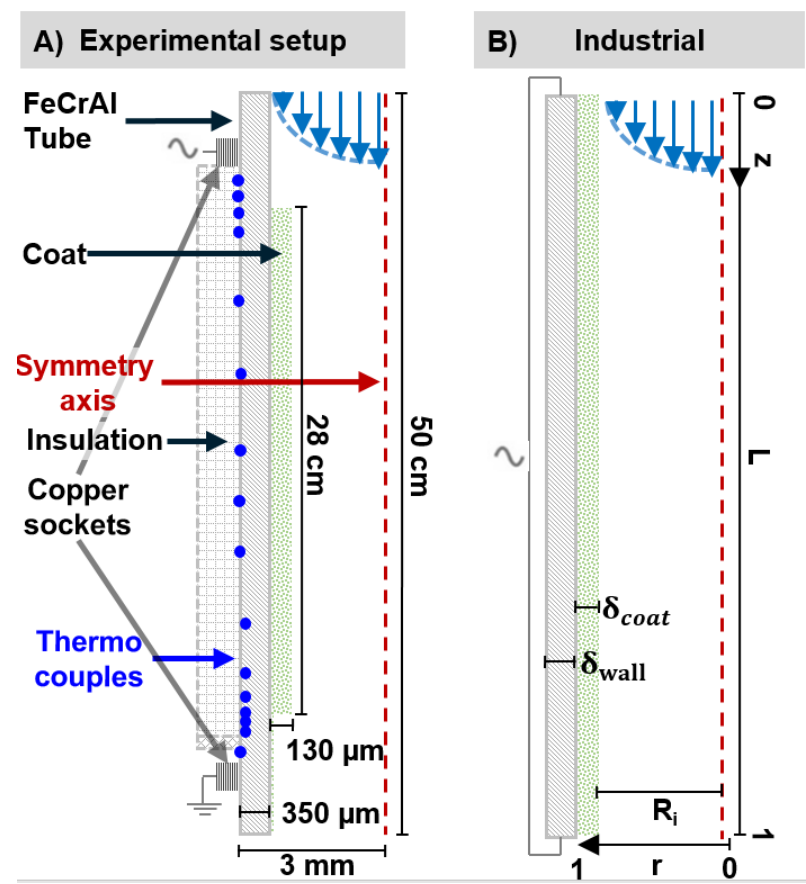

Figure 1: Reactor geometry A) Illustration of the axisymmetrical reactor geometry used for the CFD model. The indicated dimensions represent the experimental setup. The blue dots indicate positions of the spotwelded thermocouples on the reactor wall. B) Illustration of reduced channel geometry used in simulations at industrial conditions, with coat for the entire reactor length. $\delta_{\text {coat }}$ is washcoat thickness, $\delta_{\text {wall }}$ is wall thickness, and $R_{i}$ the internal radius-excluding the washcoat. $L$ is reactor tube length. $z$ and $r$ denote, respectively, vertical and radial positions, normalized against reactor length and internal radius. Note that the center $(r=0)$ is defined on the symmetry axis. The illustrations are not to scale.

\section{Model implementation}

A Computational Fluid Dynamics (CFD) model using standard equations for energy, fluid dynamics, mass transfer, and electric fields was implemented in Comsol 5.2a to obtain insight into 
parameters that could not be measured, and as a base to extrapolate towards industrially relevant conditions. ${ }^{38}$ A detailed description of the model implementation is included in the supplementary information. The modelled domains of the ambient reactor setup are shown in Figure 1A. The implemented CFD model is fitted to three parameters; thermal conductivity of insulation, preexponential catalytic activity factor, and layer thickness of residual coat towards the outlet (Fig. S2). The CFD model was fitted using a least square approach, against six experimental data sets spanning different flows and conversion. All presented data is using the best fit, evaluated based on a weighted temperature profile and methane conversion, reproducing experimental temperature data within an average error of $\pm 10^{\circ} \mathrm{C}$. Methane conversion is predicted within $\pm 1.9 \%$ (Fig. S3).

The model was extended to typical industrial conditions based on a side-fired SMR with a capacity of $50.000 \mathrm{Nm}^{3} \mathrm{H}_{2} /$ h. Ideal insulation, no electrical contact resistance, and gas properties behaving as ideal within moderate pressures are assumed for simulations at industrial conditions. The reduced geometry is shown in Fig. 1B. The ideal insulation is assumed to be a reasonable approximation, as external losses at industrial scale typically is less than $2 \%$, and integration of the heat source is expected to improve energy efficiency. ${ }^{10}$

It is stressed that these simulations assume an ideal coat, with homogeneous distribution of pores and catalytic activity.

\section{Characteristic timescales}

A typical heterogeneous catalytic reaction consists of five steps to form the product: bulk diffusion, internal diffusion, adsorption, reaction, and desorption. ${ }^{39}$ Characteristic timescale analysis is introduced in order to detect the limiting phenomena based on the timescale for the reaction steps. For an endothermic reaction, the relevant timescales are; external mass transfer, internal mass transfer, heat transfer, reaction- and contact time. ${ }^{40}$ Contact time is estimated from space-time instead of average residence time, as the flow is at all points laminar as the Reynolds number is less than 1200 , well below the transition to turbulence at $2100 .{ }^{38}$ The space-time is defined as: ${ }^{41}$

$$
\tau_{s}=\frac{z_{c}}{u_{z c}}
$$

Where $z_{c}$ is the axial position in the reactor center $(\mathrm{r}=0)$, and $u_{z c}$ is the linear velocity, evaluated at $z_{c}$. When compared to average reaction time, it is evaluated at the entrance $\left(z_{C}=0 \mathrm{~cm}\right)$. Spacetime is evaluated by the velocity at the tube center, opposed to residence time calculated as the volume divided by flow.

The external mass transport time coefficient is defined as: ${ }^{42-44}$

$$
\tau_{\text {ext }}=\frac{R_{i}^{2}}{4 D_{m}}
$$

Here $R_{i}$ is the internal radius, not including catalytic washcoat. $D_{m}$ is the averaged diffusion coefficient for methane. 
The internal mass transport time coefficient is defined as: ${ }^{45}$

$$
\tau_{\text {int }}=\frac{\delta_{\text {coat }}^{2}}{D_{e f f}}
$$

Here $\delta_{\text {coat }}$ is the coat thickness, and $D_{\text {eff }}$ the effective diffusion in the coat. ${ }^{46}$

$$
\begin{gathered}
D_{e f f}=\frac{\varepsilon}{\tau}\left(\frac{1}{D_{K}}+\frac{1}{D_{m}}\right)^{-1} \\
\tau=\varepsilon^{-1 / 2}
\end{gathered}
$$

Here $\varepsilon$ is coat porosity, $\tau$ coat tortuosity calculated by the Bruggeman correlation, $D_{m}$ the mixtureaveraged bulk diffusion coefficient, and $D_{K}$ the Knudsen diffusion, defined as: ${ }^{46}$

$$
D_{K}=\frac{\alpha}{3} \sqrt{\frac{8 R T}{\pi \bar{M}}}
$$

Where $\alpha$ is the average pore diameter, $\bar{M}$ the average molecular weight of the gas species, $R$ the gas constant, and $T$ temperature. A simple correlation for tortuosity is implemented, as different approximations of tortuosity have very little influence for moderate porosities. ${ }^{47}$

The heat transfer time coefficient is defined as: ${ }^{38}$

$$
\tau_{h t}=\frac{\delta^{2}}{\alpha}
$$

Here $\delta$ is the conduction length (wash-coat thickness for the electrified reformer), and $\alpha$ is thermal diffusivity, estimated by:

$$
\alpha=\frac{k}{\rho C_{p}}
$$

Where $k$ is thermal conductivity, $\rho$ is material density, and $C_{p}$ is specific heat capacity. The coat is at all instances limiting compared to heat transfer across the reactor wall $\left(\tau_{h t, w a l l}<\tau_{h t, \text { coat }}\right)$ (Fig. S4).

The reaction time coefficient is defined as: ${ }^{45}$

$$
\tau_{r e}=\frac{C_{C H_{4}}}{R_{C H_{4}}}
$$


Here $C_{\mathrm{CH}_{4}}$ is the methane concentration, and $R_{\mathrm{CH}_{4}}$ the overall methane consumption rate, both evaluated at the catalyst surface.

The catalyst effectiveness factor, $\eta$, is a measure of what fraction of the catalyst is utilized, by dividing the bulk rate with the ideal rate (no internal mass transport) evaluated at the surface:

$$
\eta=\frac{\int_{0}^{\delta} \mathrm{R}_{\mathrm{CH}_{4}}(\mathrm{r}) \mathrm{dr}}{\delta_{\text {coat }} \cdot \mathrm{R}_{\mathrm{CH}_{4}}^{\text {surface }}} \cdot 100 \%
$$

The effectiveness in Equation (13) is calculated at a given axial position, across the coat. For evaluation of the effectiveness factor for the entire reactor, the axial position is included in an additional integral.

\section{Results \& Discussion}

\subsection{Experimental temperature profiles}

To evaluate the performance of the electrified steam methane reforming lab reactor unit, it was operated at varying flow rates and input powers. Temperature profiles of the external reactor surface for different experimental conditions are shown in Figure 2. Between the copper socket and start of the catalytic coat $(6-11 \mathrm{~cm})$, the temperature quickly increases, as all supplied energy goes towards heating the process gas. The change in slope, followed by a linear temperature profile for the catalytic section $(11-39 \mathrm{~cm})$ is evidence of the endothermic reaction consuming the available heat. The following region $(40-44 \mathrm{~cm})$ with a second linear temperature profile indicates weak catalytic activity (Fig. S2), insufficient to keep the reaction at equilibrium, followed by rapid cooling, due to un-insulated heat exchange with the surroundings. 


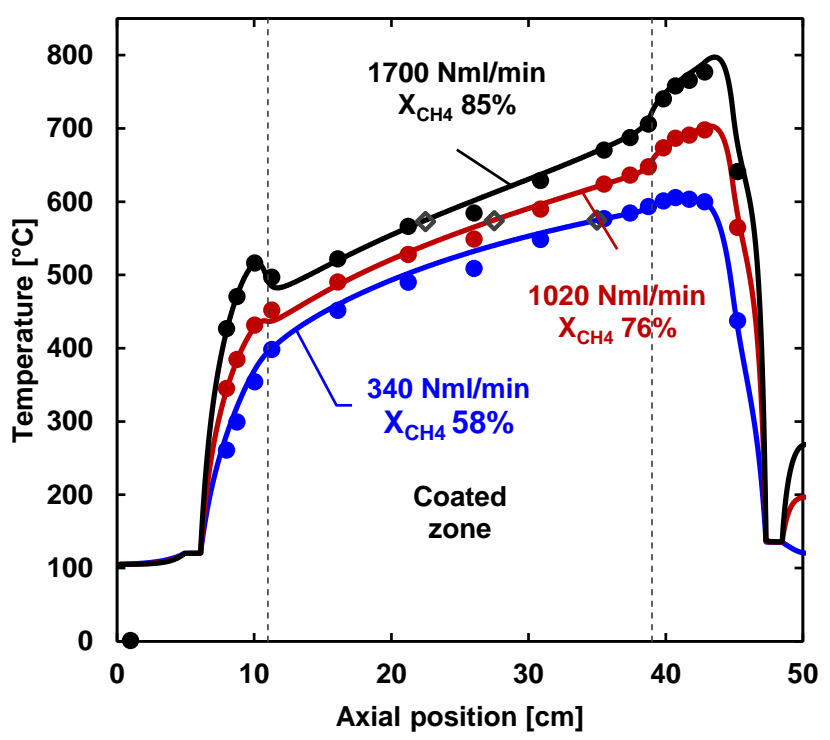

Figure 2: Temperature profiles Selected temperature profiles at different flows and conversions. Filled symbols represent measured values, solid lines the modelled profile. Open symbols indicate where the thermal gradient across the coat is evaluated in Figure 3B. Experiments performed at ambient pressure, $105{ }^{\circ} \mathrm{C}$ inlet temperature, at a steam-to carbon ratio $(S / C)=2$, with $10 \%$ hydrogen in the feed. For 340,1020, and $1700 \mathrm{Nml} / \mathrm{min}$, the average inward heat flux in the coated zone was $2.3,9.0$, and $17.6 \mathrm{~kW} / \mathrm{m}^{2}$, respectively.

The linear region of the temperature profile in the coated zone indicates the reaction is kept near equilibrium at the catalytic surface, with a near uniform heat flux (Fig. S5). Heat generation in the reactor wall is nearly constant, because the resistivity of $\mathrm{FeCrAl}$ (reactor wall) is practically independent of temperature. Consequently, the gas is heated rapidly in the non-catalytic zone (6$11 \mathrm{~cm})$. More energy is required as the flow and conversion increases, heating the gas above equilibrium in the non-catalytic zone, resulting in a drop in temperature at the beginning of the coated zone (Fig. 2). The additional latent energy of the pre-heated gas results in high reaction rates, quickly driving the reaction towards equilibrium, consuming more heat than supplied locally, causing the drop in temperature.

The three temperature profiles in Figure 2 corresponds to an average heat duty of 1.5, 5.6, and 10.5 $\mathrm{W} / \mathrm{cm}^{3}$ based on the change in enthalpy evaluated between the inlet $(6 \mathrm{~cm})$ and peak temperature $(43 \mathrm{~cm})$. The duty is comparable to large-scale industrial reformers, which typically operates between 0.5 to $7.5 \mathrm{~W} / \mathrm{cm}^{3}{ }^{3,10}$ For reference, fired micro-reactors can reach up to $65 \mathrm{~W} / \mathrm{cm}^{3}$ in the reaction zone. ${ }^{24}$

The high heat flux in large-scale reformers, and low thermal conductivity of the reactor wall and catalyst, result in steep thermal gradients in the packed bed industrial reactors (Fig. 3A). In contrast, the shorter length scales utilized in the catalytic wash-coat configuration limit thermal gradients and improve catalyst utilization. However, wash-coated reactors for strongly 
endothermic reactions are rarely seen at large scale, as it is difficult to supply sufficient heat. ${ }^{48}$ With integrated resistance heating, heat is supplied in intimate contact to the catalyst, and practically no thermal difference is observed across the catalytic wash-coat (Fig. 3B).
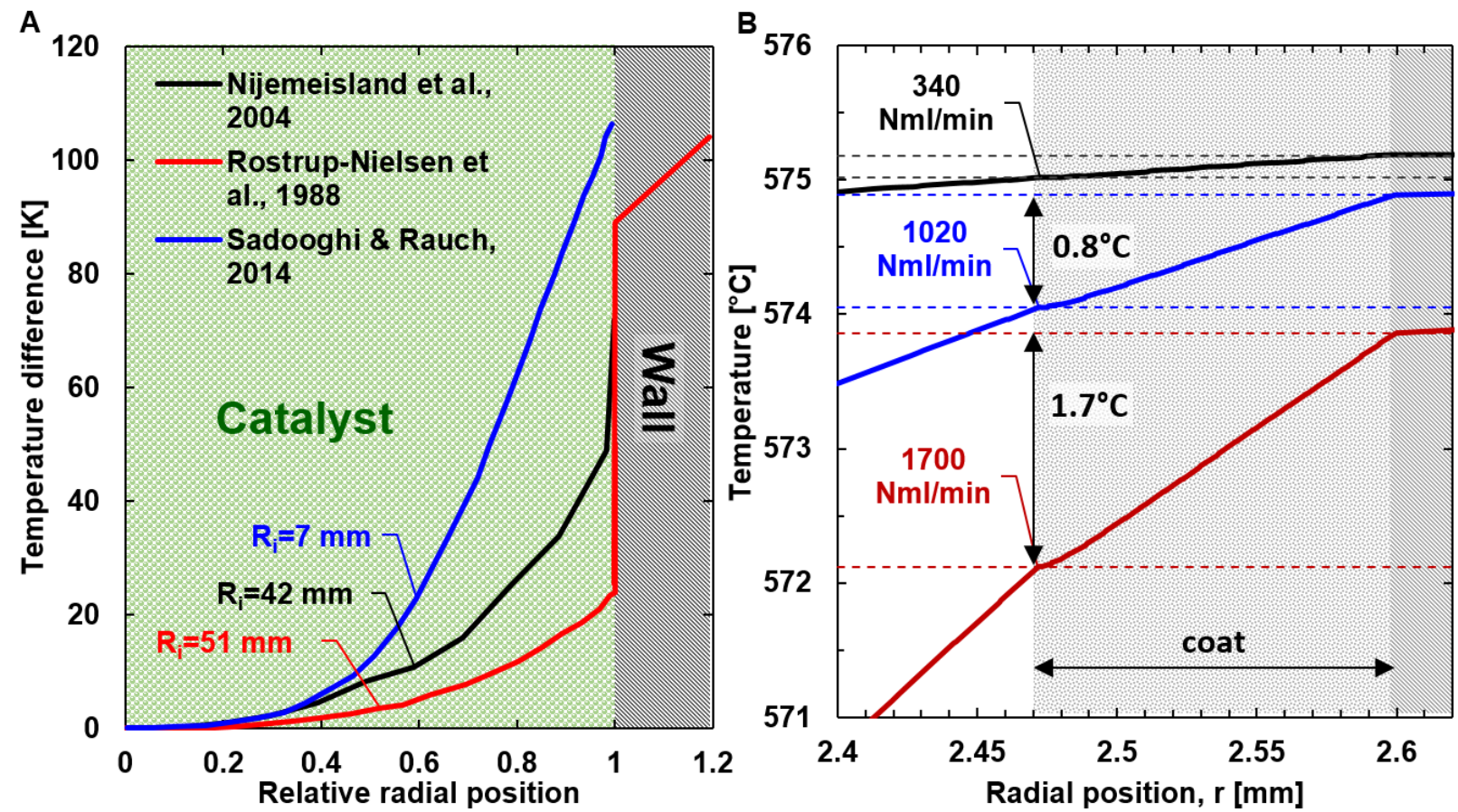

Figure 3: Temperature profile across catalyst A) Radial temperature profiles across packed bed catalyst for SMR reactors coat. Data adapted from Nijemeisland et al., 2004; Rostrup-Nielsen et al., 1988; and Sadooghi \& Rauch, 2014. ${ }^{49-51}$ The temperature difference relative to the reactor center is used, as the absolute temperature depends on operating conditions. B) Modelled temperature profiles across the catalytic washcoat. Simulation conditions: $105^{\circ} \mathrm{C}$ inlet, ambient pressure, $S / C=2$, with 10\% hydrogen in the feed. Evaluated at 35, 27.5, and $22.5 \mathrm{~cm}$ for 340, 1020, and $1700 \mathrm{Nml} / \mathrm{min}$ respectively - cf. Fig. 2.

Thermal differences exceeding $100 \mathrm{~K}$ between reactor wall and center is reported for packed bed reformers for SMR (Fig. 3A). The significant difference in limitation of the tube film layer, a zone between reactor wall and catalyst with a higher void, ${ }^{52}$ is likely related to different size and shape of catalyst pellets, and the operating conditions. As the flow rate and methane conversion increases, more power is required to drive the reaction, increasing the thermal difference across the catalytic coat, as the reaction concomitantly consumes more heat at the internal surface when not kinetically limited. However, the predicted temperature difference across the coat at the $85 \%$ methane conversion and $1700 \mathrm{Nml} / \mathrm{min}$ is less than $2^{\circ} \mathrm{C}$ (Fig. 3B). The temperature difference across the wall does not change significantly along the coated zone (Fig. S6). Moreover, there is no discernable gradient across the reactor wall (Fig. 3B), a substantial benefit compared to conventional fired reformers, where thermal difference over the reactor wall is detrimental to reactor lifetime, and limits catalyst utilization. ${ }^{5,19}$ 
Without thermal gradients, a substantial improvement of catalyst effectiveness can be achieved, exceeding $24 \%$ for the experimentally investigated configuration and conditions. ${ }^{33}$

\subsection{Scale-up to industrial conditions}

A section without catalytic activity can be used for controlled pre-heating of the feed-gas, but serves no practical purpose in an industrial setting. By coating the entire reactor length (Fig. 1B), the process gas is kept close to equilibrium along the entire reactor. Based on the experiments at ambient pressure, evidence of high accuracy for the model was verified. ${ }^{33}$ Consequently, the CFD model is extrapolated to typical industrial conditions, based on a $50.000 \mathrm{Nm}^{3} \mathrm{H}_{2} / \mathrm{h}$ side-fired SMR reformer. The most significant changes are the increased inlet temperature and outlet pressure. A temperature increase of $250^{\circ} \mathrm{C}$ is required to reach equivalent conversion as the outlet pressure is increased from ambient to 27.7 bar. Figure 4 shows the predicted temperature profile at ambient (for the coated zone) and industrial conditions, at $75 \%$ and $90 \%$ methane conversion.

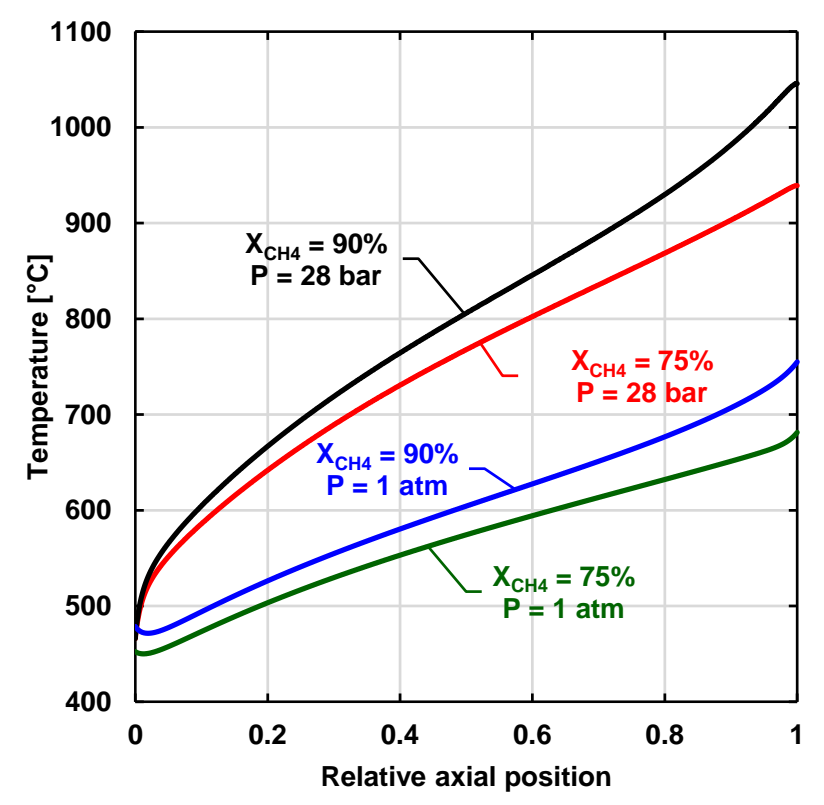

Figure 4: Temperature profiles at ambient and industrial conditions Modelled temperature profiles at ambient and industrial conditions. Profiles are only along the coated zone (i.e., positions 11-39 cm in the experimental set up, cf. Fig. 2, and the entire tube length for the highpressure profiles). Industrial conditions: $9900 \mathrm{GHSV}, 27.7 \mathrm{bar}, 465^{\circ} \mathrm{C}$ inlet, and $\mathrm{S} / \mathrm{C}=1.8$, $Q_{\text {avg }}=12.5 \& 15.1 \mathrm{~kW} / \mathrm{m}^{2}$ for 75 and $90 \%$ conversion respectively. The inlet gas composition is in equilibrium. Ambient conditions: $9900 \mathrm{GHSV}, 1 \mathrm{~atm}, 105{ }^{\circ} \mathrm{C}$ inlet, $S \mathrm{C}=2$, with $10 \%$ hydrogen in feed gas, 10 to $11.9 \mathrm{~kW} / \mathrm{m}^{2}$. All simulations are for reactor tubes with length $L=50 \mathrm{~cm}$ and inner diameter $D_{i}$ of $5.3 \mathrm{~mm}$

Similar to the experiments at ambient pressure, a steep increase in temperature is observed near the inlet, as high pressure results in low reaction rate at the inlet. A nearly linear temperature profile 
is observed along the majority of the reactor, as the reaction consumes available heat. Operating at a higher methane conversion, an increase in temperature is predicted towards the outlet, as the conversion near the catalyst surface approaches unity, kinetically limiting the reaction rate, thus consuming less heat. Without altering geometry or operating conditions, this results in a practical upper limit of achievable conversion (Fig. S3).

\subsection{Catalytic effectiveness factor}

Low catalytic effectiveness factor in packed bed reactors for endothermic processes is typically due to insufficient thermal conductivity rather than lack of kinetic activity. ${ }^{5,19,53}$ The average effectiveness factor for a conventional fired reformer is less than $3 \% .{ }^{5}$ As there is practically no thermal gradient across the wash-coated catalyst of the electrified methane reformer (Figs. 3B and S6), a catalyst effectiveness factor near $25 \%$ was achieved at the experimental setup at ambient conditions, ${ }^{33}$ ca. an order of magnitude higher than typically achieved in conventional fired reactors. ${ }^{5}$ No significant change to the effectiveness factor is expected by scaling to industrial conditions in similar geometry, as the current design is limited by mass transport, nearly independent of pressure, as both linear gas velocity and bulk diffusion is inversely proportional to pressure. ${ }^{38}$ As the catalyst effectiveness factor is evaluated by the ratio of the rate at the surface to the bulk rate, and the rate rapidly declines across the catalytic layer (Fig. S7), it is possible to increase the effectiveness factor, by decreasing the washcoat layer thickness (Fig. 5).

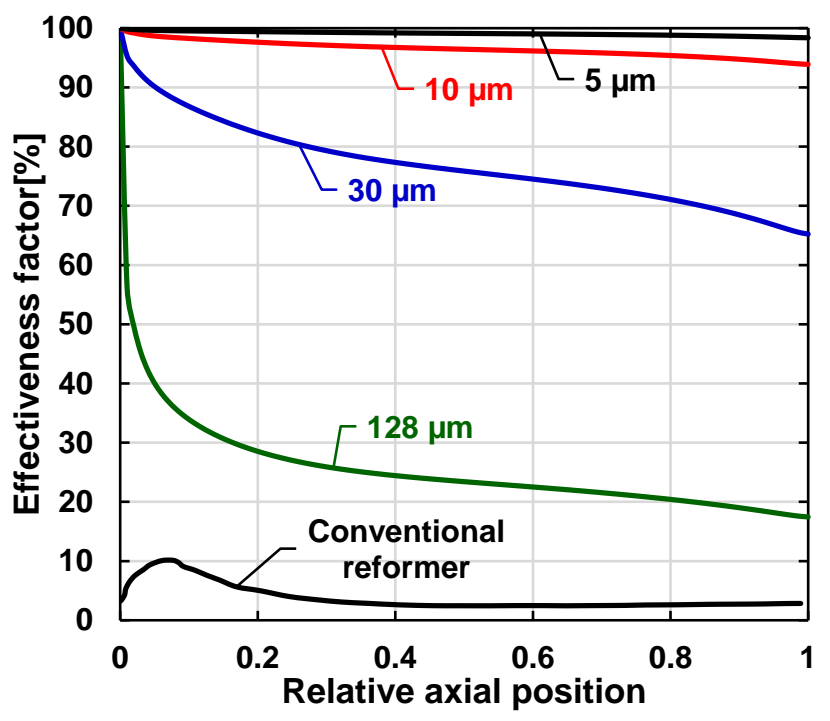

Figure 5: Catalyst effectiveness factor Axial effectiveness profiles for decreasing coat thickness. Effectiveness factor for conventional top-fired reformer adapted from Rostrup-Nielsen \& Christensen, 2011. ${ }^{5}$ Simulations at 27.7 bar, $S C=1.8,6325 h^{-1} \mathrm{GHSV}, 465{ }^{\circ} \mathrm{C}$ inlet, $Q_{\text {avg }}=7.6$ $\mathrm{kW} / \mathrm{m}^{2}$, and ca. $90 \%$ methane conversion. 
The peak in effectiveness factor near the inlet for the conventional reformer (Fig. 5) is a result of preheating the gas to around $650^{\circ} \mathrm{C}$, driving the reaction from the latent heat. In the model for the electrified reformer at industrial conditions, the gas is not preheated relative to typical outlet temperature of a pre-reformer, yielding relative low reaction rates near the inlet (Fig. S8), allowing the gas to equilibrate across the coat, resulting in a local effectiveness factor near unity. As the temperature increases, the reaction rate increases and less catalyst is required to reach equilibrium, observed in the declining effectiveness factor along the reactor (Fig. S8). Towards the outlet of the reactor, the effectiveness factor declines faster, as the methane conversion at the coat surface approaches unity due to very fast kinetics at the highly elevated temperatures (Fig. S9).

In steady state, a surplus amount of catalyst has little to no impact on conversion, as illustrated by Figure 6. However, continuous removal of the catalyst eventually leads to insufficient catalytic material to facilitate the reaction, resulting in an increasing outlet temperature and a risk of detrimental side reactions, as seen as the asymptotic behaviors at $0-10 \mu \mathrm{m}$ coat thickness in Figure 6. The increase in average effectiveness factor (Fig. 6) yields an optimum around $30 \mu \mathrm{m}$ of coat to guarantee sufficient catalytic activity (Fig. S10). This corresponds well with predictions by Mbodji et al. for a microstructured SMR reactor at short residence time. ${ }^{45}$ Further reduction leads to insufficient catalytic activity, as seen from the sharp increase in approach towards equilibrium, $\beta$, and outlet temperature.

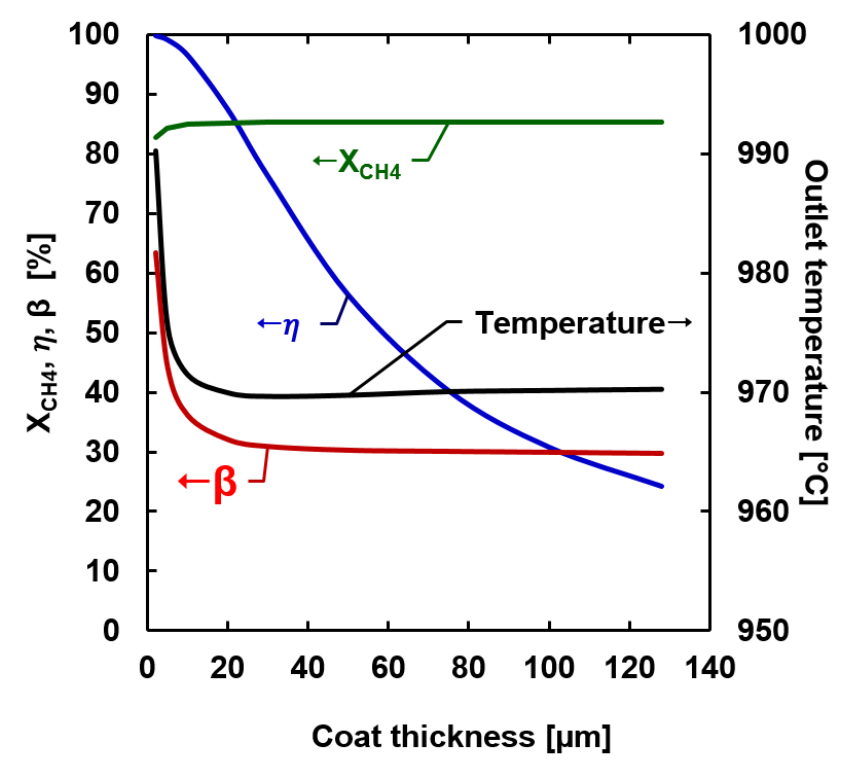

Figure 6: Influence of washcoat thickness Methane conversion ( $\left.X_{C H 4}\right)$, effectiveness factor ( $\left.\eta\right)$, approach towards equilibrium ( $\beta$ ), and outlet temperature as a function of coat thickness. Simulations at $27.7 \mathrm{bar}, \mathrm{S} / \mathrm{C}=1.8$ (Equilibrated at $465^{\circ} \mathrm{C}$ ), $Q_{\text {avg }}=7.6 \mathrm{~kW} / \mathrm{m}^{2}, 6325 \mathrm{~h}^{-1} \mathrm{GHSV}, T_{\text {inlet }}$ $=465^{\circ} \mathrm{C}$. 
From a practical perspective, very thin coats are more susceptible to loss of activity, from poisoning or loss of surface area due to sintering. ${ }^{54}$ Ideal coat layer thickness will therefore be a compromise between safety margins and optimal use of catalyst raw materials. Thicker coats will induce larger thermal gradients, increase linear gas velocity, and does not yield improved performance for a mass transfer limited reaction. While very thin coats $(<20 \mu \mathrm{m})$ provide very high catalytic effectiveness factors, the approach to equilibrium increases substantially, indicating a kinetic limit for the reaction.

Assuming stable operation at $30 \mu \mathrm{m}$ of coat, the catalyst volume can be reduced by nearly two orders of magnitude relative to a conventional reformer, resulting in an average effectiveness factor around $75 \%$.

\subsection{Characteristic timescales}

The limiting phenomena can be estimated through analysis of the characteristic timescales for the system (Eq. 6-14). The characteristic timescales for the electrified reformer are compared to a typical fired reformer in Figure 7.

A

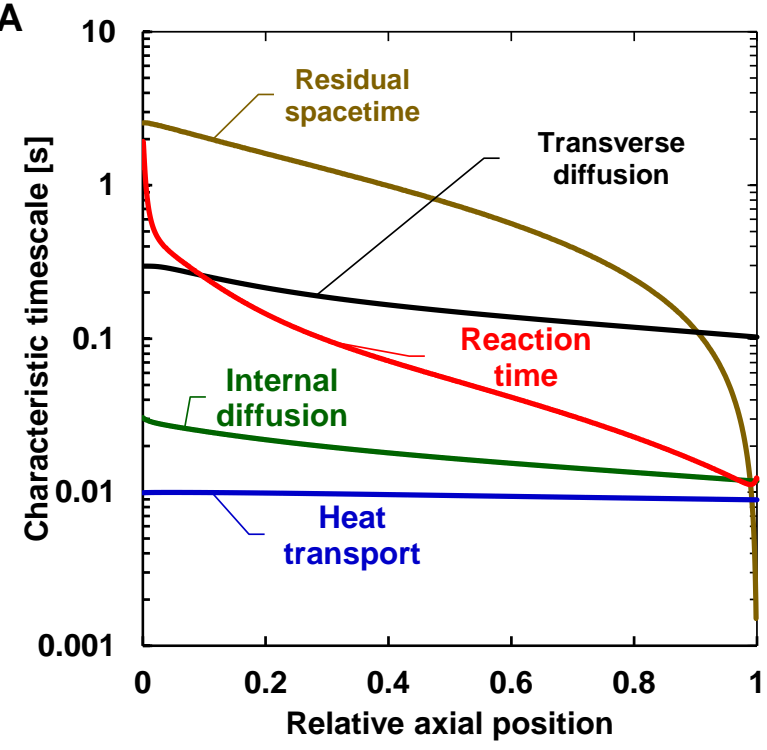

B

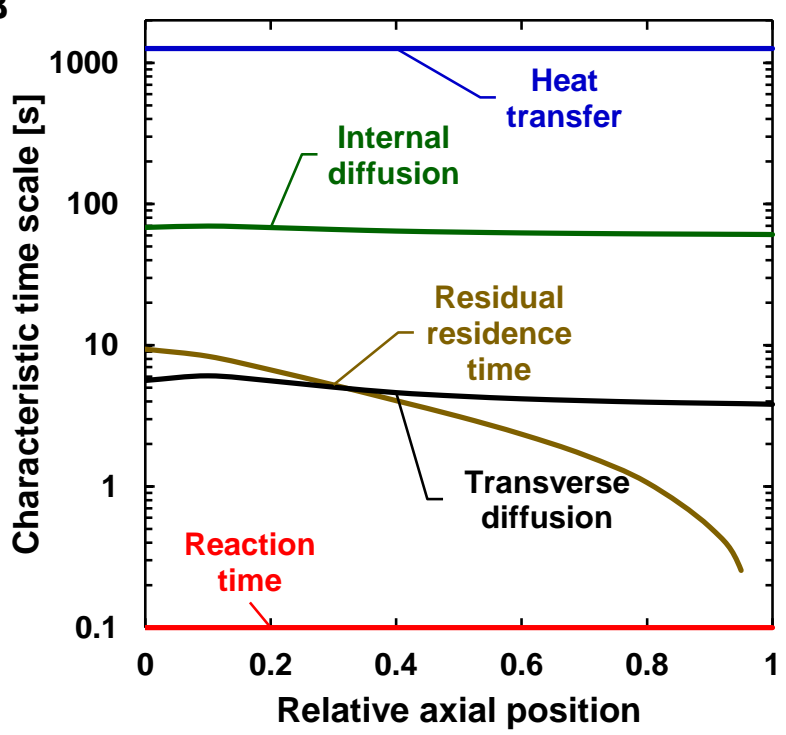

Figure 7: Characteristic timescales Axial characteristic timescales. A) Electric reformer at industrial conditions, $465^{\circ} \mathrm{C}$ inlet, 27.7 bar outlet, $S / C=1.8, Q_{\text {avg }}=7.6 \mathrm{~kW} / \mathrm{m}^{2}, 6325 \mathrm{~h}^{-1} \mathrm{GHSV}$, $5 \mathrm{~mm}$ internal diameter, and 90\% methane conversion. B) Top-fired industrial reformer with a packed bed. $829^{\circ} \mathrm{C}$ outlet, 30 bar outlet, $S / C=3.0,3500 \mathrm{GHSV}, 120 \mathrm{~mm}$ internal diameter, 71.2\% methane conversion. Estimate assumes instantaneous radiative heat transfer from furnace box to the reactor wall. Data for comparison calculated based on Kumar et al, 2017. ${ }^{10}$

Figure 7A shows that reaction kinetics is limiting the inlet of the electrified reformer, as this has the highest characteristic timescale up to a relative axial position of ca. 0.05 . However, transverse diffusion rapidly surpass the reaction rate, representing the limiting phenomena for the majority of 
the reactor. In practice, this means that the transport, e.g. diffusion, of the molecules from the center of the flow channel to the catalyst surface is the slowest mechanism of the system. Notably, heat transport is the least limiting mechanism as shown by the lowest line in Figure 7A.

For comparison, the heat transfer is by far the most limiting phenomena for a conventional fired reformer, as shown in Fig. 7B, with characteristic timescale orders of magnitude above the rest. The exceptionally high characteristic timescale for heat transport is primarily a result of low thermal conductivity and long diffusion length. As mentioned, it is an approximation of how fast a thermal perturbation is equilibrated across the domain. This is reflected in the steep thermal gradients observed in conventional reformers, which can exceed $100^{\circ} \mathrm{C}$ across the catalytic bed (Fig. 3A). ${ }^{53}$

The residual residence time in the fired reformer is below the transverse diffusion, indicating radial mixing of the gas is slow (Fig. 7B). It should be noted the transverse diffusion in Fig. 7B does not take turbulence into account, which may lower it substantially. The internal diffusion in Figure 7B is based on a $5.4 \mathrm{~mm}$ pellet, ${ }^{10}$ and supports previous observations, that performance of fired packed-bed reformers scales with catalyst surface area rather than volume. ${ }^{5}$

The reaction time in Figure $7 \mathrm{~B}$ is calculated based on average process gas composition. ${ }^{10}$ Scaled differently (Fig. S11), the reaction time displays a trend similar to the effectiveness factor (Fig. 5), indicating a substantial surplus of available catalytic activity, and that the average gas composition

is far from equilibrium, based on the kinetic model by $\mathrm{Xu} \&$ Froment. ${ }^{55} \mathrm{~A}$ reaction close to equilibrium will have a high characteristic timescale, as the approach towards equilibrium is near unity, suppressing the reaction rate.

\subsection{Influence of diffusion length scale}

Transverse diffusion represents the main limiting phenomena for the electrified reformer (as illustrated by Figure 7A). This is primarily dependent on the characteristic length scale for mass transport, which for flow in pipes is the internal diameter. As there is negligible flow in the washcoat, it is not included in the internal diameter for calculating characteristic bulk diffusion. Figure 8A shows reactor performance for different reactor length and internal diameter, operating at an average outlet temperature of $1015^{\circ} \mathrm{C}$, assuming a fixed coat $(130 \mu \mathrm{m})$ and wall thickness $(350$ $\mu \mathrm{m})$, and a constant ratio between supplied energy and methane flow. As the internal diameter is the characteristic bulk diffusion length scale, decreasing it reduce the mass transfer limitation alleviating concentration gradients across the reactor (Fig. S9). 

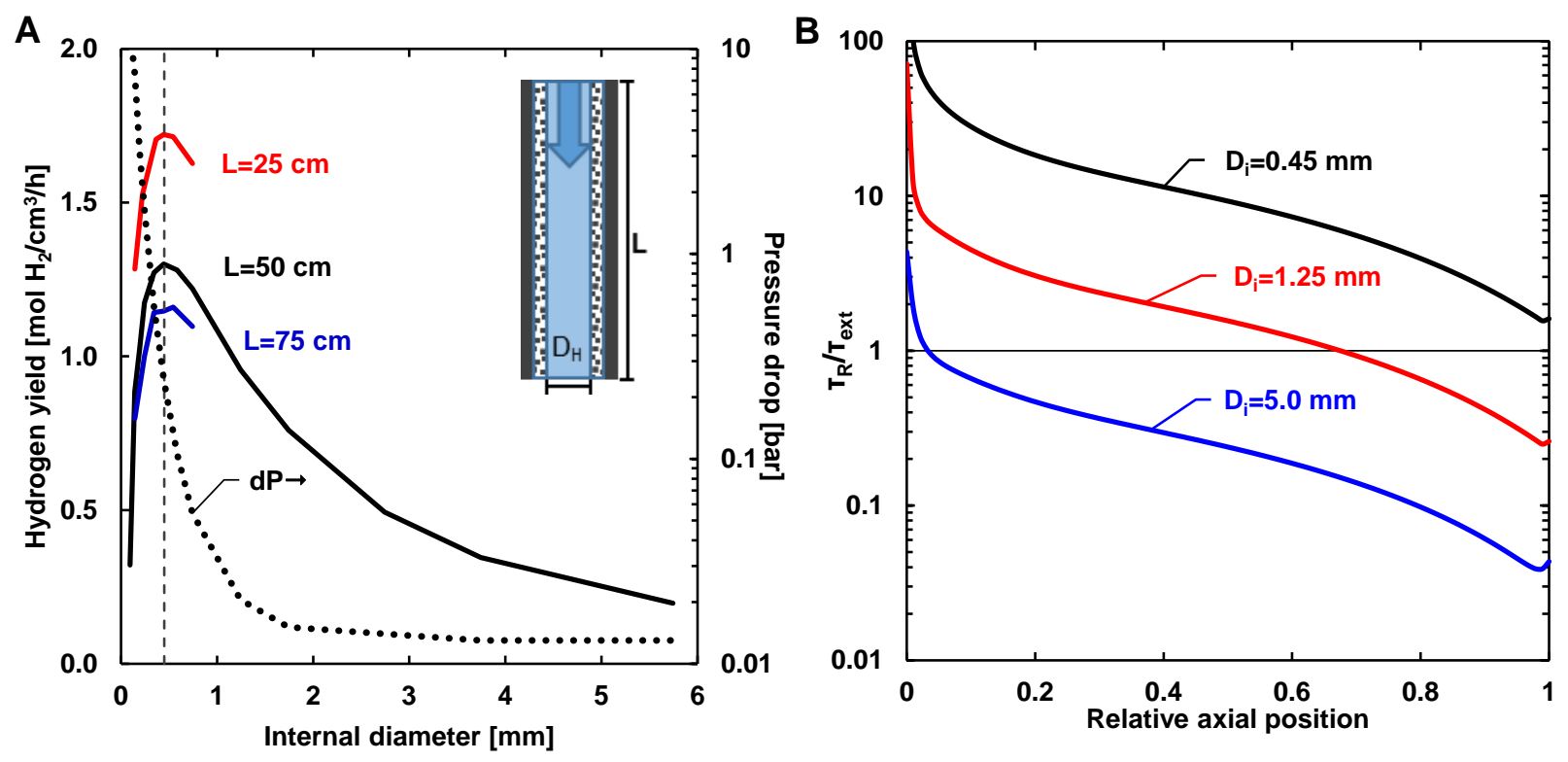

Figure 8: Influence of reactor dimensions A) Modelled hydrogen yield as a function of internal diameter and reactor length. B) Ratio of characteristic reaction time to bulk diffusion for 0.45 , 1.25 and $5.0 \mathrm{~mm}$ internal diameter (not including washcoat), at 5415 h-1, $4365 \mathrm{~h}^{-1}$, and $615 \mathrm{~h}^{-1}$ GHSV, and 23.7, 21.7 and $9.4 \mathrm{~kW}$ avg $/ \mathrm{m}^{2}$, respectively, $T_{\text {out }, \text { avg }}=1015^{\circ} \mathrm{C}, P_{\text {out }}=27.7 \mathrm{bar}, \mathrm{S} / \mathrm{C} 1.8$.

A significant increase in reactor capacity is predicted by reducing the internal diameter, but with an increasing pressure drop (Fig. 8A). For comparison, a typical fired reformer operates at ca. 0.1 $\mathrm{mol} \mathrm{H} / \mathrm{cm}^{3}$ cat $/$ h relative to the catalyst volume. ${ }^{10}$ An optimal diameter of $0.45 \mathrm{~mm}$ is predicted at the given conditions, independent of reactor length within the given range, in agreement with previous results for micro-reactor studies. ${ }^{56,57}$ The optimum is encountered where reaction time and mass transfer are balanced towards the outlet, as seen in Figure 8B. Further reduction in diameter results in a steep drop, as the space-time becomes smaller than the characteristic reaction time (Fig. S12). This is in part due to the increasing pressure drop, increasing the required temperature to facilitate the reaction (Fig. S1). Shorter reactors incur a lower pressure drop and can operate at higher flow rates per reactor volume (Figs. 8 and S13). For reactors shorter than 20 $\mathrm{cm}$, insufficient contact time results in decreasing methane conversion, and increasing risk of adverse side-effects (Fig. S13). With increasing feed flow an equivalent increase in heat flux is required to drive the reaction, resulting in a higher temperature difference across the catalytic coat (Fig. S14). It should be emphasized that the predicted temperature gradients across the coat, for reactors longer than $20 \mathrm{~cm}$, are less than $5^{\circ} \mathrm{C}$ (Fig. S15).

For an internal diameter of $5.0 \mathrm{~mm}$, performance for practically the entire reactor length is limited by mass transport as $\tau_{R} / \tau_{\text {ext }}<1$ (Fig. 8B), as also predicted in Figure 7A. At a diameter of 0.45 $\mathrm{mm}$, performance of the entire reactor length is limited by reaction kinetics rather than mass transport resulting in a higher performance due to the facile kinetics.

The pressure drop is strongly dependent on the internal diameter, and scales linearly with reactor 
length and flowrate ${ }^{38}$ Doubling reactor length and flow will require a higher average heat flux to reach equivalent conversion, as the pressure drop increases fourfold. It should be noted that the pressure drop is based on fully laminar flow in smooth tubes. The Reynolds number never exceeds 1200 , well below the transition to turbulence at $2100 .^{38}$ For practical operation, the uneven surface of the wash-coat may yield a larger pressure drop - however, still at a fraction of what is observed in typical conventional reformers. ${ }^{5,10}$

Assuming steady state, ideal coat, and Knudsen diffusion, internal diffusion will at no point be limiting, but thermal conductivity may result in small gradients unless coat thickness is reduced (Fig. S14).

With integration of an electrical heat source, the furnace is obsolete, which for a conventional reformer accounts for $98-99 \%$ of the reformer volume, not including the heat recovery from the flue gas. ${ }^{5,10,33}$ Compared to a conventional packed bed reactor, catalyst make up a relatively low fraction of the reactor volume for a wash-coated geometry. Despite the substantially lower catalyst volume, the high catalyst utilization enable smaller reactors. For the experimental reformer (Fig. 1A), the catalyst volume reduction is roughly a factor of 2 , compared to the catalyst volume of a fired reformer. At optimal conditions, the reactor volume could be reduced by a factor of ca. 15, within the given assumptions (Fig. 8A). Further reduction could potentially be attained by reduction of wash-coat and wall thickness.

While $0.45 \mathrm{~mm}$ diameter and $20 \mathrm{~cm}$ length are predicted favorable dimensions in terms of reactor performance, this does not account for practical limitations to coating procedures, electrical connections, or the cross-section area, relevant for the voltage required to supply the electrified reformer. Determining which geometry complies with practical applications regarding scale-up, construction, and operation, requires further work within more defined conditions, regarding intended scale, operating conditions, and electrical connection.

\section{Conclusions}

This study shows how the kinetic, mass- and heat transport interplay mechanism are radically changed in electrified steam methane reforming compared to current industrial standards. Hence, the electrified reformer to a large extent eliminates the constraints imposed by the current industrial standards. Utilization of wash-coated catalyst and integrated joule heating provides an effectiveness factor, which is markedly higher than industrial steam methane reforming. To some extent the catalytic effectiveness factor becomes a redundant term, as it can be engineered to desired values, and coat thickness instead will relate to catalyst lifetime. Characteristic timescale analysis reveals that heat transfer is the least limiting mechanism, the performance is instead governed by diffusion. Optimal performance based on fluid dynamic simulations favors channels below $0.5 \mathrm{~mm}$ in diameter, and high linear gas velocities, towards alleviating mass transfer limitations. Electrified steam methane reforming is a promising alternative to the current industrial 
fired reformers, resolving thermal limitations, and enabling improved utilization of hydrocarbon feed and reduced $\mathrm{CO}_{2}$ emissions.

Associated content: Implementation of the CFD model and additional figures are included in the supporting information.

Supporting information

Supporting Figures: Fig. S1-S16

Author contribution: All authors contributed substantially to this work.

Funding: This work was supported by Innovation Fund Denmark (IFD) under file no. 516000004B and research grant 9455 from Villum Fonden.

Competing interests: The authors declare no conflicts of interest.

Data and materials availability: The catalyst can be made available under a material transfer agreement for Haldor Topsoe A/S. All other data are available in the main text or the supplementary materials.

\author{
Abbreviations: \\ CFD: Computational Fluid Dynamics \\ GHSV: Gas hourly space velocity \\ S/C: Steam-to-Carbon ratioCFD: Computational Fluid Dynamics \\ SEM: Scanning Electron Microscopy \\ SMR: Steam Methane Reforming
}




\section{References:}

(1) Bhat, S. A.; Sadhukhan, J. Process Intensification Aspects for Steam Methane Reforming: An Overview. AIChe J. 2009, 55, 408.

(2) Organisation for Economic Co-operation and Development (OECD) and International Energy Agency (IEA). Tracking Industrial Energy Efficiency and $\mathrm{CO}_{2}$ Emissions. 2007.

(3) Grand View Research. Hydrogen Generation Market Size, Share \& Trends Analysis Report By Application; 2018.

(4) Le Quéré et al., Global Carbon Budget 2018. Earth Syst. Sci. Data. 2018, 10, 2141.

(5) Rostrup-Nielsen, J.; Christiansen, L. J. Concepts in Syngas Manufacture; Imperial College Press, 2011.

(6) Sehested, J. Four Challenges for Nickel Steam-Reforming Catalysts. Catal. Today 2006, $111,103$.

(7) Behnam, M.; Dixon, A. G. 3D CFD Simulations of Local Carbon Formation in Steam Methane Reforming Catalyst Particles. Int. J. Chem. React. Eng. 2017, 15, 1.

(8) Xu, J.; Froment, G. F. Methane Steam Reforming: II. Diffusional Limitations and Reactor Simulation. AIChE J. 1989, 35, 97.

(9) Elnashaie, S. S. E. H.; Abashar, M. E. E. Steam Reforming and Methanation Effectiveness Factors Using the Dusty Gas Model under Industrial Conditions. Chem. Eng. Process. 1993, 32, 177.

(10) Kumar, A.; Baldea, M.; Edgar, T. F. A Physics-Based Model for Industrial Steam-Methane Reformer Optimization with Non-Uniform Temperature Field. Comput. Chem. Eng. 2017, $105,224$.

(11) Latham, D. A.; McAuley, K. B.; Peppley, B. A.; Raybold, T. M. Mathematical Modeling of an Industrial Steam-Methane Reformer for on-Line Deployment. Fuel Process. Technol. 2011, 92, 1574.

(12) Bonaquist, D. Analysis of $\mathrm{CO}_{2}$ Emissions, Reductions, and Capture for Large-Scale Hydrogen Production Plants. A white paper, 2010.

(13) T-Raissi, A.; Block, D. L. Hydrogen: Automotive Fuel of the Future. IEEE Power Energy Mag. 2004, 2, 40.

(14) Palma, V.; Martino, M.; Meloni, E.; Ricca, A. Novel Structured Catalysts Configuration for Intensification of Steam Reforming of Methane. Int. J. Hydrogen Energy 2017, 42, 1629.

(15) Roh, H. S.; Lee, D. K.; Koo, K. Y.; Jung, U. H.; Yoon, W. L. Natural Gas Steam Reforming for Hydrogen Production over Metal Monolith Catalyst with Efficient Heat-Transfer. Int. J. Hydrogen Energy 2010, 35, 1613.

(16) Tronconi, E.; Groppi, G.; Boger, T.; Heibel, A. Monolithic Catalysts with "high 
Conductivity" Honeycomb Supports for Gas/Solid Exothermic Reactions: Characterization of the Heat-Transfer Properties. Chem. Eng. Sci. 2004, 59, 4941.

(17) Balzarotti, R.; Beretta, A.; Groppi, G.; Tronconi, E. A Comparison between Washcoated and Packed Copper Foams for the Intensification of Methane Steam Reforming. React. Chem. Eng. 2019, 4, 1387.

(18) Balakotaiah, V.; Sun, Z.; West, D. H. Autothermal Reactor Design for Catalytic Partial Oxidations. Chem. Eng. J. 2019, 374, 1403.

(19) Aasberg-Petersen, K.; Bak Hansen, J. H.; Christensen, T. S.; Dybkjaer, I.; Christensen, P. S.; Stub Nielsen, C.; Winter Madsen, S. E. L.; Rostrup-Nielsen, J. R. Technologies for Large-Scale Gas Conversion. Appl. Catal. A Gen. 2001, 221, 379.

(20) Johnsen, K.; Ryu, H. J.; Grace, J. R.; Lim, C. J. Sorption-Enhanced Steam Reforming of Methane in a Fluidized Bed Reactor with Dolomite as $\mathrm{CO}_{2}$-Acceptor. Chem. Eng. Sci. 2006, 61,1195 .

(21) Buelens, L. C.; Galvita, V. V; Poelman, H.; Detavernier, C.; Marin, G. B. Super-Dry Reforming of Methane Intensifies CO 2 Utilization via Le Chatelier's Principle. Science. 2016, 354, 449 .

(22) Ma, R.; Castro-Dominguez, B.; Mardilovich, I. P.; Dixon, A. G.; Ma, Y. H. Experimental and Simulation Studies of the Production of Renewable Hydrogen through Ethanol Steam Reforming in a Large-Scale Catalytic Membrane Reactor. Chem. Eng. J. 2016, 303, 302.

(23) Aasberg-Petersen, K.; Nielsen, C. S.; Lægsgaard Jørgensen, S. Membrane Reforming for Hydrogen. Catal. Today 1998, 46, 193.

(24) Tonkovich, A. Y.; Perry, S.; Wang, Y.; Qiu, D.; Laplante, T.; Rogers, W. A. MicroChannel Process Technology for Compact Methane Steam Reforming. Chem. Eng. Sci. 2004, 59, 4819.

(25) Karim, A.; Bravo, J.; Gorm, D.; Conant, T.; Datye, A. Comparison of Wall-Coated and Packed-Bed Reactors for Steam Reforming of Methanol. Catal. Today 2005, 110, 86.

(26) Tonkovich, A. L. Y.; Daymo, E. A. Microreaction Systems for Large-Scale Production. Microchem. Eng. Pract. 2010, 299.

(27) Cavusoglu, G.; Dallmann, F.; Lichtenberg, H.; Goldbach, A.; Dittmeyer, R.; Grunwaldt, J. D. In Situ Characterization of Catalysts and Membranes in a Microchannel under HighTemperature Water Gas Shift Reaction Conditions. J. Phys. Conf. Ser. 2016, 712.

(28) Vinum, M. G.; Almind, M. R.; Engbæk, J. S.; Vendelbo, S. B.; Hansen, M. F.; Frandsen, C.; Bendix, J.; Mortensen, P. M. Dual-Function Cobalt-Nickel Nanoparticles Tailored for High-Temperature Induction-Heated Steam Methane Reforming. Angew. Chemie - Int. Ed. 2018, 57, 10569.

(29) Almind, M. R.; Vendelbo, S. B.; Hansen, M. F.; Vinum, M. G.; Frandsen, C.; Mortensen, P. M.; Engbæk, J. S. Improving Performance of Induction-Heated Steam Methane 
Reforming. Catal. Today, 2019, 1.

(30) Mortensen, P. M.; Engbæk, J. S.; Vendelbo, S. B.; Hansen, M. F.; Østberg, M. Direct Hysteresis Heating of Catalytically Active Ni-Co Nanoparticles as Steam Reforming Catalyst. Ind. Eng. Chem. Res. 2017.

(31) Zhang, Q.; Iqbal, A.; Sakurai, M.; Kitajima, T.; Takahashi, H.; Nakaya, M.; Ootani, T.; Kameyama, H. A 2D Model for the Cylinder Methane Steam Reformer Using Electrically Heated Alumite Catalyst. 16th World Hydrog. Energy Conf. 2006.

(32) Rieks, M.; Bellinghausen, R.; Kockmann, N.; Mleczko, L. Experimental Study of Methane Dry Reforming in an Electrically Heated Reactor. Int. J. Hydrogen Energy 2015, 40, 15940.

(33) Wismann, S. T.; Engbæk, J. S.; Vendelbo, S. B.; Bendixen, F. B.; Eriksen, W. L.; Aasbergpetersen, K.; Frandsen, C.; Chorkendorff, I.; Mortensen, P. M. Electrified Methane Reforming: A Compact Approach to Greener Industrial Hydrogen Production. Science, 2019, 364, 756 .

(34) Lazard. Lazard's Levelized Cost of Energy; 2017.

(35) International Energy Agency (IEA), World Energy Outlook 2017.

(36) Govender, S.; Friedrich, H. Monoliths: A Review of the Basics, Preparation Methods and Their Relevance to Oxidation. Catalysts 2017, 7 , 62.

(37) Rothenberg, G. Heterogeneous Catalysis. In Catalysis: Concepts and Green Applications; Wiley-VCH Verlag GmbH \& Co. KGaA: Weinheim, Germany, 2008; 127.

(38) Bird, R. B.; Stewart, W. E.; Lightfoot, E. N. Transport Phenomena, 2nd ed.; Wiley, 2002.

(39) Chorkendorff, I.; Niemantsverdriet, J. W. Concepts of Modern Catalysis and Kinetics, Wiley, 2007.

(40) Ashraf, M. A.; Sanz, O.; Montes, M.; Specchia, S. Insights into the Effect of Catalyst Loading on Methane Steam Reforming and Controlling Regime for Metallic Catalytic Monoliths. Int. J. Hydrogen Energy 2018, 43, 11778.

(41) Kockmann, N. Transport Phenomena in Micro Process Engineering; Springer Berlin Heidelberg, 2008.

(42) Bhatia, D.; McCabe, R. W.; Harold, M. P.; Balakotaiah, V. Experimental and Kinetic Study of NO Oxidation on Model Pt Catalysts. J. Catal. 2009, 266, 106.

(43) Metkar, P. S.; Salazar, N.; Muncrief, R.; Balakotaiah, V.; Harold, M. P. Selective Catalytic Reduction of NO with NH 3 on Iron Zeolite Monolithic Catalysts: Steady-State and Transient Kinetics. Appl. Catal. B Environ. 2011, 104, 110.

(44) Joshi, S. Y.; Harold, M. P.; Balakotaiah, V. On the Use of Internal Mass Transfer Coefficients in Modeling of Diffusion and Reaction in Catalytic Monoliths. Chem. Eng. Sci. 2009, 64, 4976. 
(45) Mbodji, M.; Commenge, J. M.; Falk, L. Preliminary Design and Simulation of a Microstructured Reactor for Production of Synthesis Gas by Steam Methane Reforming. Chem. Eng. Res. Des. 2014, 92, 1728.

(46) Nield, D. A.; Bejan, A. Convection in Porous Media; Springer, 2013.

(47) Shen, L.; Chen, Z. Critical Review of the Impact of Tortuosity on Diffusion. Chem. Eng. Sci. 2007, 62, 3748 .

(48) Baharudin, L.; Watson, M. J. Monolithic Substrate Support Catalyst Design Considerations for Steam Methane Reforming Operation. Rev. Chem. Eng. 2017, $0,1$.

(49) Nijemeisland, M.; Dixon, A. G.; Stitt, E. H. Catalyst Design by CFD for Heat Transfer and Reaction in Steam Reforming. Chem. Eng. Sci. 2004, 59, 5185.

(50) Rostrup-Nielsen, J. R.; Christiansen, L. J.; Bak Hansen, J. H. Activity of Steam Reforming Catalysts. Appl. Catal. 1988, 43, 287.

(51) Sadooghi, P.; Rauch, R. Experimental and Modeling Study of Catalytic Steam Reforming of Methane Mixture with Propylene in a Packed Bed Reactor. Int. J. Heat Mass Transf. 2014, 78, 515 .

(52) Geb, D.; Zhou, F.; Catton, I. Internal Heat Transfer Coefficient Determination in a Packed Bed From the Transient Response Due to Solid Phase Induction Heating. J. Heat Transfer 2012, 134 .

(53) Wehinger, G. D.; Kraume, M.; Berg, V.; Korup, O.; Mette, K.; Schlögl, R.; Behrens, M.; Horn, R. Investigating Dry Reforming of Methane with Spatial Reactor Profiles and Particle-Resolved CFD Simulations. AlChe J. 2016, 62, 4436.

(54) Simonsen, S. B.; Sehested, J.; Helveg, S.; Chorkendorff, I.; Dahl, S.; Skoglundh, M. Direct Observations of Oxygen-Induced Platinum Nanoparticle Ripening Studied by in Situ TEM. J. Am. Chem. Soc. 2010, 132, 7968..

(55) Xu, J. G.; Froment, G. F. Methane Steam Reforming, Methanation and Water-Gas Shift .1. Intrinsic Kinetics. Aiche J. 1989, 35, 88.

(56) Zhai, X.; Ding, S.; Chang, Y.; Jin, Y.; Cheng, Y. CFD Simulation with Detailed Chemistry of Steam Reforming of Methane for Hydrogen Production in an Integrated Micro-Reactor. Int. J. Hydrogen Energy 2010, 35, 5383.

(57) Mbodji, M.; Commenge, J. M.; Falk, L.; Di Marco, D.; Rossignol, F.; Prost, L.; Valentin, S.; Joly, R.; Del-Gallo, P. Steam Methane Reforming Reaction Process Intensification by Using a Millistructured Reactor: Experimental Setup and Model Validation for Global Kinetic Reaction Rate Estimation. Chem. Eng. J. 2012, 207-208, 871. 